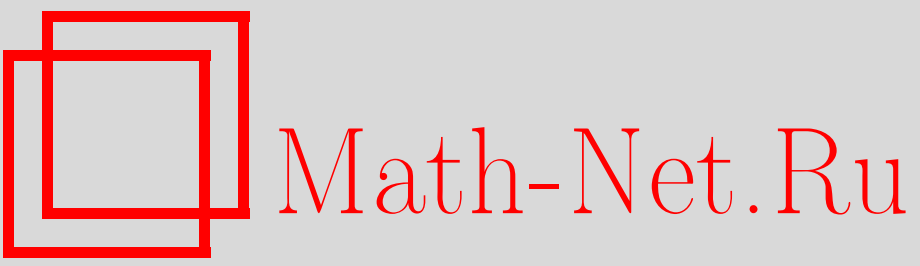

П. А. Яськов, Сильная сходимость кратных сумм неортогональных случайных величин, Теория вероятн. и ее примен., 2010, том 55, выпуск 2, 382-386

DOI: https://doi.org/10.4213/tvp4210

Использование Общероссийского математического портала Math-Net.Ru подразумевает, что вы прочитали и согласны с пользовательским соглашением

http://www . mathnet.ru/rus/agreement

Параметры загрузки:

IP: 18.207 .199 .55

26 апреля 2023 г., 15:24:34 
8. Петров В. В. Суммы независимых случайных величин. М.: Наука, 1985.

9. Клебанов Л. Б., Мания Г. М., Меламед И. А. Одна задача В. М. Золотарева и аналоги безгранично делимых и устойчивых распределений в схеме суммирования случайного числа случайных величин. - Теория вероятн. и ее примен., 1984, т. 29 , в. 4 , с. $757-760$.

10. Круглов В.М., Королев В.Ю. Предельные теоремы для случайных сумм. М.: Изд-во Моск. ун-та, 1990, 269 с.

11. Sato K.-I. Lévy Processes and Infinitely Divisible Distributions. Cambridge: Cambridge Univ. Press, 1999, 486 p.

12. Сеибнев M. С. Асимптотика безгранично делимых распределений в $\mathbf{R}^{n}$. - Труды Ин-та математики СО АН СССР, 1989, т. 13, с. 100-116.

13. Сенета E. Правильно меняющиеся функции. М.: Наука, 1985, 141 с.

14. Феллер В. Введение в теорию вероятностей и ее приложения, т. 2. М.: Мир, 1984, $752 \mathrm{c}$.

15. Якымив А.Л. Вероятностные приложения тауберовых теорем. М.: Физматлит, $2005,256 \mathrm{c}$.

Поступила в редакцию 22.IX.2008

(c) 2010 г.

ЯСЬКОВ П. А.*

\section{СИЛЬНАЯ СХОДИМОСТЬ КРАТНЫХ СУММ НЕОРТОГОНАЛЬНЫХ СЛУЧАЙНЫХ ВЕЛИЧИН ${ }^{1)}$}

Получены новые достаточные условия п.н. сходимости кратных рядов из неортогональных случайных величин и новый вариант усиленного закона больших чисел. Данные условия описаны в виде ограничений на вторые моменты, а также на верхние грани средних попарных произведений рассматриваемых величин. Наши теоремы улучшают как хорошо известные результаты для квазистационарных полей, так и недавние, связанные с обобщением свойства квазистационарности.

Ключевые слова и фразы: случайное поле, усиленный закон больших чисел, сходимость рядов почти наверное, зависимые случайные величины.

1. Введение. В настоящей статье рассматривается вопрос об асимптотическом поведении кратных сумм, образованных элементами случайного поля определенного класса. Точнее говоря, исследуются условия применимости усиленного закона больших чисел, а также сходимости кратных рядов с вероятностью единица. Упомянутый класс весьма широк, в частности, он содержит квазистационарные поля.

Данному вопросу посвящено много работ. Исчерпывающие ответы получены для следующих типов случайных полей: составленных из независимых одинаково распределенных величин (см. [1] и [2]), ортогональных (см. [3] и [4]), однородных (см. [5]), квазистационарных (см. [6]). Ограничения общего вида, не преполагающие какой-либо структуры зависимости, рассмотрены в [7]. Наша статья мотивирована недавними работами [8] и [9], где допускается некоторое отклонение от свойства квазистационарности.

Многие результаты о квазистационарных случайных величинах получаются с помощью максимальных неравенств Морица (см. [10]). В нашем случае эти неравенства

* Московский государственный университет им. М.В. Ломоносова, Ленинские горы, 119991 Москва, ГСП-1, Россия; e-mail: yaskov_pavel@mail.ru

1) Работа выполнена при финансовой поддержке РФФИ, грант № 07-01-00373. 
оказываются неприменимыми. Поэтому мы устанавливаем их новые варианты. Для доказательства наших неравенств мы применияем простые и эффективные средства (ср. с [10]). При выводе основных теорем мы используем технику стандартную для многопараметрического случая.

Для более точных формулировок введем ряд обозначений. Положим $x^{+}=x \vee 0$, $\log x=\log _{2}(x \vee 2),\langle\mathbf{x}\rangle=\prod_{i=1}^{d}\left(x_{i} \vee 1\right)$ и $f(\mathbf{x})=\left(f\left(x_{1}\right), \ldots, f\left(x_{d}\right)\right)$ для $x \in \mathbf{R}_{+}, \mathbf{x}=$ $\left(x_{1}, \ldots, x_{d}\right) \in \mathbf{R}_{+}^{d}$ и $f: \mathbf{R}_{+} \rightarrow \mathbf{R}$. Зададим на $\mathbf{N}_{0}^{d}$ покомпонентную операцию сложения и естественный частичный порядок. Пусть $\mathbf{0}=(0, \ldots, 0) \in \mathbf{N}_{0}^{d}, \mathbf{1}=(1, \ldots, 1) \in \mathbf{N}_{0}^{d}$. Если $\mathbf{m}, \mathbf{n} \in \mathbf{N}_{0}^{d}$, то также определим $\mathbf{m n}=\left(m_{1} n_{1}, \ldots, m_{d} n_{d}\right),\|\mathbf{n}\|=\max \left\{n_{1}, \ldots, n_{d}\right\}$, $[\mathbf{m}, \mathbf{n}]=\left\{\mathbf{k} \in \mathbf{N}_{0}^{d} \mid \mathbf{m} \leqslant \mathbf{k} \leqslant \mathbf{n}\right\}$ и $\sum_{\mathbf{k}=\mathbf{m}}^{\mathbf{n}}=\sum_{\mathbf{k} \in[\mathbf{m}, \mathbf{n}]}$, где сумма по пустому множеству считается равной нулю. Запись $\mathbf{n} \rightarrow \infty$ означает, что $\min \left\{n_{1}, \ldots, n_{d}\right\} \rightarrow \infty$.

Пусть $X_{\mathbf{n}}, \mathbf{n} \in \mathbf{N}^{d}$ - случайные величины с конечным моментом второго порядка. Если $\mathbf{E} X_{\mathbf{m}} X_{\mathbf{n}}=0$ при $\mathbf{m} \neq \mathbf{n}$ и константы $a_{\mathbf{n}}, \mathbf{n} \in \mathbf{N}^{d}$, таковы, что

$$
\sum_{\mathbf{n} \geqslant \mathbf{1}} a_{\mathbf{n}}^{2} \mathbf{E} X_{\mathbf{n}}^{2}\langle\log \mathbf{n}\rangle^{2}<\infty
$$

то, согласно (1) расширенной теореме Меньшова-Радемахера (см., например, [4]), ряд

$$
\sum_{\mathbf{n} \geqslant \mathbf{1}} a_{\mathbf{n}} X_{\mathbf{n}} \text { сходится п.н. }
$$

При $d=1$ в [11] приведено обобщение этой теоремы, в котором вместо ортогональных величин $X_{\mathbf{n}}$ берутся квазистационарные, т.е. такие, что $\phi_{\mathbf{k}}=$ $\sup _{\mathbf{m}, \mathbf{n}:|\mathbf{m}-\mathbf{n}|=\mathbf{k}}\left|\mathbf{E} X_{\mathbf{m}} X_{\mathbf{n}}\right|$ конечно при всех $\mathbf{k} \in \mathbf{N}_{0}^{d}$. В [8] и [9], наряду с конечностью $\phi_{\mathbf{k}}$ для $\mathbf{k} \in \mathbf{N}_{0}^{d} \backslash\{\mathbf{0}\}$, допускается рост $\mathbf{E} X_{\mathbf{n}}^{2}$. Подобный случай исследуется и в настоящей статье.

Итак, в данной работе результаты [11] о п.н. сходимости случайных последовательностей распространяются на случайные поля, удовлетворяющие (1). В [11] используются более ограничительные по сравнению с (1) условия вида $\sup _{n \geqslant 1} \mathbf{E} X_{n}^{2}=1$ и $\sum_{n \geqslant 1} W(n) a_{n}^{2}$, где $W(n), n \in \mathbf{N}$, обладают следующим свойством: $\log ^{2} n=O(W(n))$ при $n \rightarrow \infty$. Нетрудно видеть, что наши теоремы улучшают [8], [9], а также часть результатов [6].

2. Формулировки основных результатов. Нам понадобится ряд обозначений. Пусть $\rho_{\mathbf{k}}=\sup _{\mathbf{m}, \mathbf{n}:|\mathbf{m}-\mathbf{n}|=\mathbf{k}}\left(\mathbf{E} X_{\mathbf{m}} X_{\mathbf{n}}\right)^{+}$при $\mathbf{k} \in \mathbf{N}_{0}^{d} \backslash\{\mathbf{0}\}$ и $\rho_{\mathbf{0}}=0$. Следуя [11], введем

$$
w(\mathbf{n})=\sum_{\mathbf{k}=\mathbf{0}}^{\mathbf{n}} \rho_{\mathbf{k}} .
$$

При таком задании величины $w(\mathbf{n})$ не убывают по $\mathbf{n}$ относительно частичного порядка на $\mathbf{N}_{0}^{d}$. Напомним, что запись $\varepsilon \in[\mathbf{0}, \mathbf{1}]$ означает, что вектор $\varepsilon$ состоит из нулей и единиц. Положим для $\varepsilon \in[\mathbf{0}, \mathbf{1}]$ и $\mathbf{n} \in \mathbf{N}_{0}^{d}$

$$
W(\mathbf{n}, \boldsymbol{\varepsilon})^{1 / 2}=\sum_{\mathbf{m}=\boldsymbol{\varepsilon}[\log \mathbf{n}]}^{[\log \mathbf{n}]} w\left(2^{\mathbf{m}}\right)^{1 / 2},
$$

где $[x]$ - целая часть $x \in \mathbf{R}$. В силу монотонности $w$, очевидно, имеем $W(\mathbf{n}, \boldsymbol{\varepsilon})=$ $O\left(w(\mathbf{n})\langle\log \boldsymbol{\delta} \mathbf{n}\rangle^{2}\right)$ при $\boldsymbol{\delta}=\mathbf{1}-\boldsymbol{\varepsilon}$, когда $\|\mathbf{n}\| \rightarrow \infty$. Если к тому же $\liminf _{\|\mathbf{n}\| \rightarrow \infty} w(\mathbf{n})>0$, то $\langle\log \boldsymbol{\delta} \mathbf{n}\rangle^{2}=O(W(\mathbf{n}, \boldsymbol{\varepsilon}))$. Определим $\Phi_{c}$ как класс неубывающих функции $\varphi: \mathbf{R}_{+} \rightarrow$ $\mathbf{R}_{+}$, для которых ряд $\sum_{n \geqslant 0} 1 / \varphi(n)$ сходится.

Сформулируем главные результаты нашей работы.

Теорема 1. Пусть выполнено (1) и $a_{\mathbf{n}} \geqslant 0$ при всех $\mathbf{n}$. Тогда для справедливости (2) достаточно, чтобы для некоторой $\varphi \in \Phi_{c}$ при всех $\varepsilon \in[0,1]$

$$
\sum_{\mathbf{n} \geqslant \mathbf{1}} W(\mathbf{n}, \boldsymbol{\varepsilon})\langle\varphi(\log \boldsymbol{\varepsilon} \mathbf{n})\rangle a_{\mathbf{n}}^{2}<\infty .
$$


Теорема 2. Пусть $\sum_{\mathbf{n} \geqslant 0} \sum_{\mathbf{k}=2^{\mathbf{n}}}^{2^{\mathbf{n}+\mathbf{1}}} \mathbf{E} X_{\mathbf{k}}^{2}\langle\mathbf{n}\rangle^{2} / b_{2^{\mathbf{n}}}^{2}<\infty$, где $b_{\mathbf{n}}, \mathbf{n} \in \mathbf{N}^{d}-$ maкие положительные постояннье, что $b_{\mathbf{n}}$ не убывает по $\mathbf{n} u$

$$
\sum_{\mathbf{m}: 2^{\mathbf{m}} \geqslant \mathbf{n}} 1 / b_{2 \mathbf{m}}^{2}=O\left(\langle\log \mathbf{n}\rangle^{2} / b_{\mathbf{n}}^{2}\right), \quad\|\mathbf{n}\| \rightarrow \infty
$$

Предположим, ито для некоторой $\varphi \in \Phi_{c}$ при всех $\boldsymbol{\varepsilon} \in[\mathbf{0}, \mathbf{1}] \backslash\{\mathbf{1}\}$ имеет место (5), где $a_{\mathbf{n}}$ заменено на $1 / b_{\mathbf{n}}$. Если при этом $W(\mathbf{n}, \mathbf{1})\langle\varphi(\log \mathbf{n})\rangle / b_{\mathbf{n}}^{2}$ не возрастает по $\mathbf{n}$, mo

$$
\lim _{\|\mathbf{n}\| \rightarrow \infty} \frac{\sum_{\mathbf{k}=\mathbf{1}}^{\mathbf{n}} X_{\mathbf{k}}}{b_{\mathbf{n}}}=0 \text { и.н. }
$$

Заметим, что если в качестве $\rho_{\mathbf{k}}$ взять величины $\phi_{\mathbf{k}}$ (см. введение), то ограничение вида $\inf _{\mathbf{n}} a_{\mathbf{n}} \geqslant \mathbf{0}$ в теореме 1 можно убрать. Кроме того, в случае, когда $\varphi(2 x) \leqslant C \varphi(x)$ при всех $x \in \mathbf{R}_{+}$для некоторого $C>0$, вместо монотонности $W(\mathbf{n}, \mathbf{1})\langle\varphi(\log \mathbf{n})\rangle / b_{\mathbf{n}}^{2}$ в условии теоремы 2 можно потребовать невозрастание $W(\mathbf{n}, \mathbf{1}) / b_{\mathbf{n}}^{2}$.

Доказательство теорем 1 и 2 основано на следующих моментных неравенствах.

Лемма 1. Пусть $S_{\mathbf{m}, \mathbf{n}}=\sum_{\mathbf{k}=m}^{\mathbf{m}+\mathbf{n}-1} a_{\mathbf{k}} X_{\mathbf{k}}$. Тогда

$$
\mathbf{E} S_{\mathbf{m}, \mathbf{n}}^{2} \leqslant \sum_{\mathbf{k}=\mathbf{m}}^{\mathbf{m}+\mathbf{n}-\mathbf{1}} a_{\mathbf{k}}^{2} \mathbf{E} X_{\mathbf{k}}^{2}+2^{d} w(\mathbf{n}) \sum_{\mathbf{k}=\mathbf{m}}^{\mathbf{m}+\mathbf{n}-\mathbf{1}} a_{\mathbf{k}}^{2}
$$

где $ш$ определено в (3).

Лемма 2. Пусть $M_{\mathbf{n}}(\boldsymbol{\delta})=\max _{(\mathbf{1}-\boldsymbol{\delta}) 2^{\mathbf{n}}+\boldsymbol{\delta} \leqslant \mathbf{k} \leqslant 2^{\mathbf{n}}}\left|S_{2^{\mathbf{n}}, \mathbf{k}}\right|$, где $\boldsymbol{\delta} \in[\mathbf{0}, \mathbf{1}] u S_{2^{\mathbf{n}}, \mathbf{k}}$ введены в формулировке леммы 1. Тогда

$$
\mathbf{E} M_{\mathbf{n}}^{2}(\boldsymbol{\delta}) \leqslant 2\langle\boldsymbol{\delta} \mathbf{n}\rangle^{2} \sum_{\mathbf{k}=2^{\mathbf{n}}}^{2^{\mathbf{n}+\mathbf{1}}-\mathbf{1}} a_{\mathbf{k}}^{2} \mathbf{E} X_{\mathbf{k}}^{2}+2^{d+1} W\left(2^{\mathbf{n}}, \boldsymbol{\varepsilon}\right) \sum_{\mathbf{k}=2^{\mathbf{n}}}^{2^{\mathbf{n}+\mathbf{1}}-\mathbf{1}} a_{\mathbf{k}}^{2},
$$

где $\boldsymbol{\varepsilon}=\mathbf{1}-\boldsymbol{\delta}$, а $W$ задается формулой (4).

Если $\sup _{\mathbf{k}} \mathbf{E} X_{\mathbf{k}}^{2} \leqslant 1$, то (8) вытекает из максимального неравенства Морица (см. [10, следствие 3]), которое получается с помощью индукции по $\mathbf{n}$ и $d$. В случае, когда ограничений на $\mathbf{E} X_{\mathbf{k}}^{2}$ нет, такая индукция не позволяет вывести (8), в отличие от прямого способа оценки $\mathbf{E} M_{\mathbf{n}}^{2}(\boldsymbol{\delta})$.

3. Доказательства. Лемма 1 вытекает из определения $\rho_{\mathbf{k}}$ и неравенства Коши. Установим лемму 2. Рассмотрим вначале случай $\boldsymbol{\delta}=\mathbf{1}$. Обозначим

$$
S_{U}=\sum_{\mathbf{i} \in U} a_{\mathbf{i}+2^{\mathbf{n}}} X_{\mathbf{i}+2^{\mathbf{n}}} \quad \text { при } \quad U \subset\left[\mathbf{0}, 2^{\mathbf{n}}-\mathbf{1}\right], \quad I(\mathbf{k}, \mathbf{m})=\left[(\mathbf{k}-\mathbf{1}) 2^{\mathbf{m}}, \mathbf{k} 2^{\mathbf{m}}-\mathbf{1}\right] .
$$

Очевидно, что $I\left(\mathbf{k}_{1}, \mathbf{m}\right) \cap I\left(\mathbf{k}_{2}, \mathbf{m}\right)=\varnothing$, если $\mathbf{k}_{1} \neq \mathbf{k}_{2}$, и $\cup_{\mathbf{k}=\mathbf{1}}^{2^{\mathbf{n}} \mathbf{m}} I(\mathbf{k}, \mathbf{m})=\left[\mathbf{0}, 2^{\mathbf{n}}-\mathbf{1}\right]$. Имеем

$$
M_{\mathbf{n}}(\mathbf{1}) \leqslant \sum_{\mathbf{m}=\mathbf{0}}^{\mathbf{n}} \max _{\mathbf{1} \leqslant \mathbf{k} \leqslant 2^{\mathbf{n}-\mathbf{m}}}\left|S_{I(\mathbf{k}, \mathbf{m})}\right|,
$$

откуда по неравенству Коши-Буняковского

$$
\mathbf{E} M_{\mathbf{n}}^{2}(\mathbf{1}) \leqslant \sum_{\mathbf{m}=\mathbf{0}}^{\mathbf{n}}\left(\mathbf{E} M_{\mathbf{n}}^{2}(\mathbf{1})\right)^{1 / 2}\left(\mathbf{E} \max _{\mathbf{1} \leqslant \mathbf{k} \leqslant 2^{\mathbf{n}-\mathbf{m}}} S_{I(\mathbf{k}, \mathbf{m})}^{2}\right)^{1 / 2} .
$$

Согласно лемме 1 справедлива следующая оценка:

$$
\mathbf{E} \max _{\mathbf{1} \leqslant \mathbf{k} \leqslant 2^{\mathbf{n}-\mathbf{m}}} S_{I(\mathbf{k}, \mathbf{m})}^{2} \leqslant \sum_{\mathbf{k}=1}^{2^{\mathbf{n}-\mathbf{m}}} \mathbf{E} S_{I(\mathbf{k}, \mathbf{m})}^{2} \leqslant \sum_{\mathbf{i}=2^{\mathbf{n}}}^{2^{\mathbf{n}+\mathbf{1}}-\mathbf{1}} a_{\mathbf{i}}^{2} \mathbf{E} X_{\mathbf{i}}^{2}+2^{d} w\left(2^{\mathbf{m}}\right) \sum_{\mathbf{i}=2^{\mathbf{n}}}^{2^{\mathbf{n}+\mathbf{1}}-\mathbf{1}} a_{\mathbf{i}}^{2} .
$$


В итоге, применяя неравенство Минковского, получаем

$$
\left(\mathbf{E} M_{\mathbf{n}}^{2}(\mathbf{1})\right)^{1 / 2} \leqslant\langle\mathbf{n}\rangle\left(\sum_{\mathbf{i}=2^{\mathbf{n}}}^{2^{\mathbf{n}+\mathbf{1}}-\mathbf{1}} a_{\mathbf{i}}^{2} \mathbf{E} X_{\mathbf{i}}^{2}\right)^{1 / 2}+\sum_{\mathbf{m}=\mathbf{0}}^{\mathbf{n}} w\left(2^{\mathbf{m}}\right)^{1 / 2} \cdot\left(2^{d} \sum_{\mathbf{i}=2^{\mathbf{n}}}^{2^{\mathbf{n}+\mathbf{1}}-\mathbf{1}} a_{\mathbf{i}}^{2}\right)^{1 / 2} .
$$

Отсюда и из неравенства Коши вытекает (8). Случай $\boldsymbol{\delta} \neq \mathbf{1}$ рассматривается аналогично, если в качестве $I(\mathbf{k}, \mathbf{m})$ взять $\left[(\mathbf{k}-1) 2^{\mathbf{m}+(\mathbf{1}-\boldsymbol{\delta}) \mathbf{n}}, \mathbf{k} 2^{\mathbf{m}+(\mathbf{1}-\boldsymbol{\delta}) \mathbf{n}}-\mathbf{1}\right]$ для $\mathbf{m} \leqslant \boldsymbol{\delta} \mathbf{n}$. Лемма 2 установлена.

Перейдем к доказательству теоремы 1. Оно состоит из двух шагов. Первый это проверка того, что

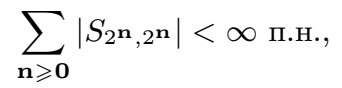

второй — вывод соотношения

$$
T_{\mathbf{n}}=\max _{2^{\mathbf{n}} \leqslant \mathbf{k} \leqslant 2^{\mathbf{n}+1}-\mathbf{1}}\left|S_{\mathbf{k}}-S_{2^{\mathbf{n}}-\mathbf{1}}\right| \rightarrow 0 \text { п.н., } \quad \mathbf{n} \rightarrow \infty,
$$

где $S_{\mathbf{k}}=\sum_{\mathbf{i}=\mathbf{1}}^{\mathbf{k}} a_{\mathbf{i}} X_{\mathbf{i}}$. Заметим, что из (9) следует существование предела $\lim _{\mathbf{n} \rightarrow \infty} S_{2^{\mathbf{n}}-\mathbf{1}}$ п.н. Согласно теореме Б. Леви сходимость $\sum_{\mathbf{n} \geqslant \mathbf{0}} \mathbf{E}\left|S_{2^{\mathbf{n}}, 2^{\mathbf{n}}}\right|$ ведет к (9). Полагая здесь и далее $C_{\varphi}=\sum_{n \geqslant 0} 1 / \varphi(n)+1$, по неравенству Коши-Буняковского имеем

$$
\sum_{\mathbf{n} \geqslant \mathbf{0}} \mathbf{E}\left|S_{2^{\mathbf{n}}, 2^{\mathbf{n}}}\right|=\sum_{\mathbf{n} \geqslant \mathbf{0}} \mathbf{E} M_{\mathbf{n}}(\mathbf{0}) \leqslant C_{\varphi}^{d}\left(\sum_{\mathbf{n} \geqslant \mathbf{0}}\langle\varphi(\mathbf{n})\rangle \mathbf{E} M_{\mathbf{n}}^{2}(\mathbf{0})\right)^{1 / 2} .
$$

Без ограничения общности считаем, что $\varphi(x) \leqslant x^{2} \vee 1$ при $x \in \mathbf{R}_{+}$. Тогда, учитывая монотонность $w$, применяя (1), (5) и лемму 2 , заключаем, что

$$
\sum_{\mathbf{n} \geqslant \mathbf{0}}\langle\varphi(\mathbf{n})\rangle \mathbf{E} M_{\mathbf{n}}^{2}(\mathbf{0}) \leqslant \sum_{\mathbf{k} \geqslant \mathbf{1}}\left(a_{\mathbf{k}}^{2} \mathbf{E} X_{\mathbf{k}}^{2}\langle\log \mathbf{k}\rangle^{2}+2^{d+1} W(\mathbf{k}, \mathbf{1})\langle\varphi(\log \mathbf{k})\rangle a_{\mathbf{k}}^{2}\right)<\infty
$$

Как и в [6], для вывода (10) используем элементарную оценку вида

$$
T_{\mathbf{n}} \leqslant \sum_{\boldsymbol{\delta} \in[\mathbf{0}, \mathbf{1}], \boldsymbol{\delta} \neq \mathbf{0}} \sum_{\mathbf{m}=\boldsymbol{\delta} \mathbf{n}}^{\mathbf{n}} M_{\mathbf{m}}(\boldsymbol{\delta}) .
$$

Фиксируем произвольное $\boldsymbol{\delta} \in[\mathbf{0}, \mathbf{1}], \boldsymbol{\delta} \neq \mathbf{0}$. Положим $\boldsymbol{\varepsilon}=\mathbf{1}-\boldsymbol{\delta}$. Имеем

$$
\sum_{\mathbf{m}=\boldsymbol{\delta} \mathbf{n}}^{\mathbf{n}} M_{\mathbf{m}}(\boldsymbol{\delta}) \leqslant \sum_{\mathbf{m} \geqslant \mathbf{0}, \boldsymbol{\delta} \mathbf{m}=\mathbf{0}} M_{\mathbf{m}+\boldsymbol{\delta} \mathbf{n}}(\boldsymbol{\delta})=Z_{\mathbf{n}}(\boldsymbol{\delta})
$$

Вследствие неравенства Коши-Буняковского, $\mathbf{E} Z_{\mathbf{n}}(\boldsymbol{\delta})^{2} \leqslant C_{\varphi}^{d} A_{\mathbf{n}}(\boldsymbol{\delta})$, где

$$
A_{\mathbf{n}}(\boldsymbol{\delta})=\sum_{\mathbf{m} \geqslant \mathbf{0}, \boldsymbol{\delta} \mathbf{m}=\mathbf{0}}\langle\varphi(\mathbf{m})\rangle \mathbf{E} M_{\mathbf{m}+\boldsymbol{\delta} \mathbf{n}}(\boldsymbol{\delta})^{2}
$$

Далее, поскольку $\langle\varphi(\mathbf{m})\rangle\langle\boldsymbol{\delta} \mathbf{n}\rangle^{2} \leqslant\langle\mathbf{m}+\boldsymbol{\delta} \mathbf{n}\rangle^{2}$ при $\boldsymbol{\delta} \mathbf{m}=\mathbf{0}$, то лемма 2 влечет

$$
A_{\mathbf{n}}(\boldsymbol{\delta}) \leqslant \sum_{\mathbf{k}: \boldsymbol{\delta} 2^{\mathbf{n}} \leqslant \boldsymbol{\delta} \mathbf{k} \leqslant \boldsymbol{\delta}\left(2^{\mathbf{n}+1}-\mathbf{1}\right)}\left(\langle\log \mathbf{k}\rangle^{2} a_{\mathbf{k}}^{2} \mathbf{E} X_{\mathbf{k}}^{2}+2^{d+1} W(\mathbf{k}, \boldsymbol{\varepsilon})\langle\varphi(\log \varepsilon \mathbf{k})\rangle a_{\mathbf{k}}^{2}\right)<\infty,
$$

откуда, принимая во внимение (1) и (5), находим, что ряд $\sum_{\mathbf{i} \geqslant 0,} \boldsymbol{\delta} \mathbf{i = \mathbf { i }} \mathbf{E} Z_{\mathbf{i}}^{2}(\boldsymbol{\delta})$ сходится. Последнее (в силу равенства $Z_{\mathbf{n}}(\boldsymbol{\delta})=Z_{\boldsymbol{\delta} \mathbf{n}}(\boldsymbol{\delta})$ и леммы Бореля-Кантелли) означает, что $\lim _{\|\mathbf{n}\| \rightarrow \infty} Z_{\mathbf{n}}(\boldsymbol{\delta})=0$ п.н. Следовательно,

$$
\lim _{\mathbf{n} \rightarrow \infty} \sum_{\mathbf{m}=\boldsymbol{\delta} \mathbf{n}}^{\mathbf{n}} M_{\mathbf{m}}(\boldsymbol{\delta})=0 \text { п.н., } \quad \boldsymbol{\delta} \in[\mathbf{0}, \mathbf{1}], \quad \boldsymbol{\delta} \neq \mathbf{0} .
$$


Учитывая соотношения (11) и (12), а также то, что векторов $\boldsymbol{\delta} \in[\mathbf{0 , 1}]$ конечное число, приходим к (10). Это завершает доказательство теоремы 1.

Обратимся к теореме 2. Положим $a_{\mathbf{k}}=1$ в определениях $S_{2^{\mathbf{n}}-\mathbf{1}}, S_{\mathbf{m}, \mathbf{n}}$, $T_{\mathbf{n}}$ и $M_{\mathbf{n}}(\boldsymbol{\delta})$. Используя условия теоремы и лемму 1 , нетрудно показать, что $\sum_{\mathbf{n} \geqslant 0} \mathbf{E} S_{2^{n_{-1}}}^{2} / b_{2^{n_{-1}}}^{2}<\infty$. Поэтому

$$
\lim _{\|\mathbf{n}\| \rightarrow \infty} S_{2^{\mathbf{n}_{-1}}} / b_{2^{\mathbf{n}_{-1}}}=0 \text { п.н. }
$$

Поступая так же, как при выводе $(12)$, с учетом монотонности $W(\mathbf{n}, \mathbf{1})\langle\varphi(\log \mathbf{n})\rangle / b_{\mathbf{n}}^{2}$ видим, что

$$
\lim _{\|\mathbf{n}\| \rightarrow \infty} Z_{\mathbf{n}}^{\prime}(\boldsymbol{\delta})=0 \quad \text { и } \quad Z_{\mathbf{n}}^{\prime}(\boldsymbol{\delta})<\infty \text { п.н., } \quad \boldsymbol{\delta} \in[\mathbf{0}, \mathbf{1}], \boldsymbol{\delta} \neq \mathbf{0},
$$

где $Z_{\mathbf{n}}^{\prime}(\boldsymbol{\delta})=\sum_{\mathbf{m} \geqslant \mathbf{0}, \boldsymbol{\delta} \mathbf{m}=\mathbf{0}} M_{\mathbf{m}+\boldsymbol{\delta} \mathbf{n}}(\boldsymbol{\delta}) / b_{2} \mathbf{m}+\delta \mathbf{n}$. Аналогично [6], в силу (11) имеем

$$
\frac{T_{\mathbf{n}}}{b_{2^{\mathbf{n}}}} \leqslant \sum_{\boldsymbol{\delta} \in[\mathbf{0}, \mathbf{1}], \boldsymbol{\delta} \neq \mathbf{0}} \sum_{\mathbf{m} \geqslant \mathbf{0}, \boldsymbol{\delta} \mathbf{m}=\mathbf{0}} B_{\mathbf{m}, \mathbf{n}}(\boldsymbol{\delta}) \frac{M_{\mathbf{m}+\boldsymbol{\delta} \mathbf{n}}(\boldsymbol{\delta})}{b_{2^{\mathbf{m}}+\delta \mathbf{n}}},
$$

где $B_{\mathbf{m}, \mathbf{n}}(\boldsymbol{\delta})=b_{2} \mathbf{m}+\delta \mathbf{n} / b_{2} \mathbf{n}$ при $\mathbf{m} \leqslant(\mathbf{1}-\boldsymbol{\delta}) \mathbf{n}$ и $B_{\mathbf{m}, \mathbf{n}}(\boldsymbol{\delta})=0$ в остальных случаях. Поскольку $b_{\mathbf{n}}$ не убывает по $\mathbf{n}$ и $\lim _{\|\mathbf{n}\| \rightarrow \infty} b_{\mathbf{n}}=\infty$ (вытекает из (6)), то $B_{\mathbf{m}, \mathbf{n}}(\boldsymbol{\delta}) \leqslant$ 1 и $B_{\mathbf{m}, \mathbf{n}}(\boldsymbol{\delta}) \rightarrow 0$, когда $\boldsymbol{\delta}, \boldsymbol{\delta} \mathbf{n}$ и $\mathbf{m}$ фиксированы, а $\|(\mathbf{1}-\boldsymbol{\delta}) \mathbf{n}\| \rightarrow \infty$. Теперь из соотношений (14) и (15) легко вывести, что $\lim _{\|\mathbf{n}\| \rightarrow \infty} T_{\mathbf{n}} / b_{2} \mathbf{n}=0$ п.н. Последнее равенство вместе с (13) дают утверждение теоремы.

Автор благодарен своему научному руководителю А. В. Булинскому за постоянное внимание и поддержку, а также признателен А. П. Шашкину за ценные замечания.

\section{СПИСОК ЛИТЕРАТУРЫ}

1. Фролов А.Н. Об асимптотическом поведении приращений случайных полей. Зап. научн. сем. ПОМИ, 2003, т. 298, с. 191-207.

2. Клесов О. И. Сходимость почти наверное кратных рядов независимых случайных величин. - Теория вероятн. и ее примен., 1995 , т. 40, в. 1, с. $68-83$.

3. Klesov $O$. On the order of growth of orthogonal random fields. - Anal. Math., 2003, v. 29 , № 1, p. 15-28.

4. Andrienko V. A. Rate of approximation by rectangular partial sums of double orthogonal series. - Anal. Math., 1996, v. 22, № 4, p. 243-266.

5. Гапошкин В.Ф. Многопараметрический усиленный закон больших чисел для однородных случайных полей. - Успехи матем. наук, 1981, т. 36, с. 197-198.

6. Moricz F. Strong laws of large numbers for quasi-stationary random fields. - Z Wahrscheinlichkeitstheor. verw. Geb., 1980, v. 51, № 3, p. 249-268.

7. Klesov O., Fazecas I., Nozaly C., Tomacs T. Strong Laws of Large Numbers for Sequences and Fields. - Theory Stoch. Proc., 1999, v. 5(21), № 3-4, p. 91-104.

8. Hu T.-C., Rosalsky A., Volodin A.I. On convergence properties of sums of dependent random variables under second moment and covariance restrictions. - Statist. Probab. Lett., 2008, v. 78, № 14, p. 1999-2005.

9. Sung S. H. Maximal inequalities for dependent random variables and applications. J. Ineq. Appl., 2008, v. 2008, Article ID 598319, doi:10.1155/2008/598319.

10. Moricz F. A general maximal inequality of the rectangular partial sums of multiple series. - Acta Math. Hungar., 1983, v. 41, № 3-4, p. 337-346.

11. Серфлинг P. Об усиленном законе больших чисел и близких результатах для квазистационарных последовательностей. - Теория вероятн. и ее примен., 1980, т. 25 , в. 1 , с. $190-194$. 